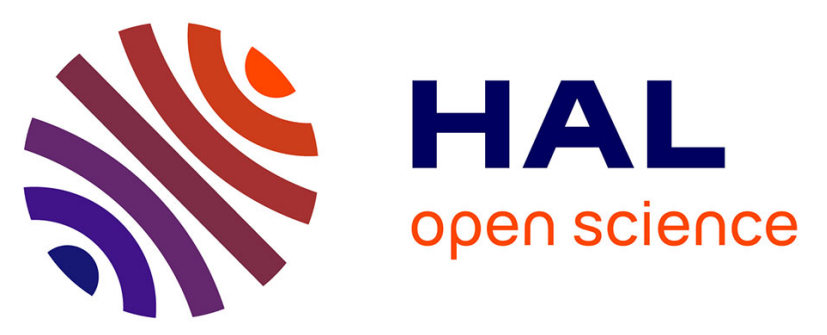

\title{
Synthesis, Structural and Optical Characterization of CdTeSe/ZnSe and CdTeSe/ZnTe Core/Shell Ternary Quantum Dots for Potential Application in Solar Cells
}

Le Xuan Hung, Pham Nam Thang, Hoang van Nong, Nguyen Hai Yen, Vu Đuc Chinh, Le van Vu, Nguyen Thi Thuc Hien, Willy Daney de Marcillac, Phan Ngoc Hong, Nguyen Thu Loan, et al.

\section{To cite this version:}

Le Xuan Hung, Pham Nam Thang, Hoang van Nong, Nguyen Hai Yen, Vu Đuc Chinh, et al.. Synthesis, Structural and Optical Characterization of CdTeSe/ZnSe and CdTeSe/ZnTe Core/Shell Ternary Quantum Dots for Potential Application in Solar Cells. Journal of Electronic Materials, 2016, pp.1-7. 10.1007/s11664-016-4578-2 . hal-01325440

\section{HAL Id: hal-01325440 \\ https: / hal.sorbonne-universite.fr/hal-01325440}

Submitted on 2 Jun 2016

HAL is a multi-disciplinary open access archive for the deposit and dissemination of scientific research documents, whether they are published or not. The documents may come from teaching and research institutions in France or abroad, or from public or private research centers.
L'archive ouverte pluridisciplinaire HAL, est destinée au dépôt et à la diffusion de documents scientifiques de niveau recherche, publiés ou non, émanant des établissements d'enseignement et de recherche français ou étrangers, des laboratoires publics ou privés. 


\title{
REMA-06
}

\section{Synthesis, structural and optical characterizations of CdTeSe/ZnSe and CdTeSe/ZnTe core/shell ternary quantum dots for potential application in solar cells}

\author{
Le Xuan Hung ${ }^{1}$, Pham Nam Thang ${ }^{2}$, Hoang Van Nong ${ }^{2}$, Nguyen Hai Yen ${ }^{2}, \mathrm{Vu}$ Đuc Chinh ${ }^{2}$, Le Van Vu ${ }^{3}$, \\ Nguyen Thi Thuc Hien ${ }^{1}$, Willy Daney de Marcillac ${ }^{4}$, Phan Ngoc Hong ${ }^{2}$, Nguyen Thu Loan ${ }^{4,2}$, Catherine Schwob ${ }^{4}$, \\ Agnès Maître ${ }^{4}$, Nguyen Quang Liem ${ }^{2}$, Paul Bénalloul ${ }^{4}$, Laurent Coolen ${ }^{4}$, Pham Thu Nga ${ }^{1,2}$ \\ ${ }^{1}$ Institute of Research and Development, Duy Tan University, Danang, Vietnam \\ ${ }^{2}$ Institute of Materials Science, Vietnam Academy of Science and Technology, \\ 18 Hoang Quoc Viet Road, Cau giay Dist., Hanoi, Vietnam \\ ${ }^{3}$ Center for Materials Science, University of Natural Science, VNU, Hanoi, Vietnam \\ ${ }^{4}$ Sorbonne Universités, UPMC Univ Paris 06, UMR 7588, Institut de NanoSciences de Paris (INSP), Paris F-75005, France
}

\begin{abstract}
This work presents the results on the fabrication, structural and optical properties of CdTeSe/ZnTe nML and CdTeSe/ZnSe nML ( $\mathrm{n}=0,1,2,4$ and 6 being the nominal shell monolayer thickness) ternary alloyed core/shell quantum dots (QDs). Transmission electron microscopy has been used to observe the shape and size of the QDs. These QDs crystallize at the zinc-blende phase. Raman scattering has been used to characterizethe CdTeSe QDs alloy composition in the fabrication and coating process. The Raman spectrum of CdTeSe QDs, in the frequency range from $100-300 \mathrm{~cm}^{-1}$, is a composite band with two peaks at $160 \mathrm{~cm}^{-1}$ and $192 \mathrm{~cm}^{-1}$. When the thickness of the ZnTe shell is $4 \mathrm{ML}$, the peak of the Raman spectrum only appears at $160 \mathrm{~cm}^{-1}$. For the ZnSe $4 \mathrm{ML}$ shell, the peak only appears at $\sim 200 \mathrm{~cm}^{-1}$. This shows that the nature of CdTeSe QDs is either CdTe-rich or CdSe-rich depending on each shell sample. The shell thickness of $2 \mathrm{ML}$ does not change the ternary core QDs' crystalline phase. The absorption and photoluminescence (PL) spectra show that the absorption and emission bands can be shifted to $900 \mathrm{~nm}$, depending on each ternary alloyed QD core/shell sample. This NIR spectrum regions is suitable for applications in solar cells.
\end{abstract}

Keywords: alloyed quantum dots, CdTeSe core/shell ternary QDs, Raman spectra, PL spectra.

\section{Introduction}

Quantum dots (QDs) with photoluminescence (PL) emission in the near-infared (NIR) range (700 - $900 \mathrm{~nm}$ ) have been the subject of many studies in the context of in-vivo imaging or semiconductor quantum dots sensitized solar cells. While CdSe (bulk band gap $1.74 \mathrm{eV}$ ) has been used tocover large parts of the visible spectrum, CdTe (bulk band gap $1.43 \mathrm{eV}$ ) provides access to NIR wavelengths. Moreover, the synthesis of alloy CdTeSe QDs allows more degrees of freedom by combining the confinement effects of the QDs with the alloying effects. First ternary CdTeSe QDs were reported by Bailey and Nie in 2003 [1]. Since then, emission up to 800 or even $900 \mathrm{~nm}$ has been reported, with a non-linear relation between the alloy composition and the absorption/emission energies. The growth of a higher-bandgap shell in order to improve QD stability and quantum yield has been the subject of few reports for CdTeSeQDs. Pons et al. reported about NIR-emitting CdTeSe/CdZnS core/shell QDs [2], or CdTeSe/CdZnS in [3], CdTeSe/ZnS [4-7]. Recently H. Zhou et al. reported the synthesis of multishell CdTeSe/ZnSe/ZnS QDs [8]. However, the number of publications concerning the coating of CdTeSe QD with ZnTe and ZnSe is still limited.

To address a novel method for fabricating QDs with NIR PL, more efforts to other preparation methods of QDs have been taken in our group. Here, we present in details a method for fabricating alloy CdTeSe QDs with different concentrations from unexpensive chemicals with low toxicity and facile synthesis. The CdTeSe/ZnTe and CdTeSe/ZnSe core-shell QDs can show PL emission which can reach $900 \mathrm{~nm}$. Detailed studies on vibration and optical characteristics of ternary alloyed QDs are also discussed in this paper.

\section{Experimental}

\subsection{Materials}

We used the following reagents (from Aldrich): cadmium acetate dihydrate $\left(\mathrm{Cd}(\mathrm{Ac})_{2} .2 \mathrm{H}_{2} \mathrm{O}, 99.9 \%\right)$, elemental selenium powder (Se, 99.99\%), elemental tellurium powder (Te, 99.99\%) as $\mathrm{Cd}$, Se and Te sources, respectly,a oleic acide (OA, 90\%) and oleylamine (OLA,90\%) as surface ligands, 1-octadecene (ODE, 90\%)and trioctylphosphine (TOP, 90\%) as the reaction medium, zinc acetate $(\mathrm{Zn}(\mathrm{Ac}) 2,99.9 \%)$ as $\mathrm{Zn}$ source for the shell preparation. All chemicals were used without further purification.

\subsection{Synthesis Method}

CdTeSe cores were prepared following amodified method described in [9-14]. Core-shell alloy quantum dots were prepared according to a modified SILAR protocol that has been previously published [13]. To carry out the fabrication of CdTeSe QDs with core/shell structure CdTeSe/ZnSe and CdTeSe/ZnTe, we followed three steps. First is to prepare precursors, then CdTeSe cores, and finally coating the QD cores with ZnSe and ZnTe shells with different thickness counted by monolayer 
(ML) $\mathrm{n}$ from $\mathrm{n}=1,2,4$ to $6 \mathrm{ML}$ ( $\mathrm{n}$ is the nominal thickness: we calculated the amounts of shell precursors to introduce into the solution in order to have stoichiometric proportions to the concentration of core QDs, depending on the core size estimated from TEM).

\section{*Precursor preparation}

In this study, we have fabricated $1 \mathrm{mmol}$ CdTeSe quantum dots (QDs) in OLA-ODE medium with two different molar ratios Cd: Te: $\mathrm{Se}=1: 1.8: 1.8$, close to the ratio used in our recent publication [13] and 10:1:1, as used in [11, 14]. Different results were obtained depending on the molar ratio. Below are the process and weight for ratio 10:1:1. The fabrication process consists of the following steps:

The Cd precursor

This fabrication method was revised from recent publications [9-12], but after many experiments, we have establisheda new method that requires a reduced amount of TOP as compared to [2], while in [9] only ODE is used, but the volume used to dissolve cadmium acetate is large, thus it is disadvantageous for the fabrication of QDs later on. The details of the method are as followings:

Weigh $1.33 \mathrm{~g}$ cadmium acetate dihydrate (corresponding to $\mathrm{Cd}$ :Te:Se $=10: 1: 1$ ), put into a three-neck flask. Then, pour in the flask $1.6 \mathrm{ml} \mathrm{OA}$ and $75 \mathrm{ml}$ ODE. The flask is vacuumed to remove air and run through by $\mathrm{N}_{2}$ gas. Afterwards, use the vigorous magnetic stirrer to remove air and increase the air to $120^{\circ} \mathrm{C}$. The process always takes place in $\mathrm{N}_{2}$ gas environment, and stirring is kept stable for 2 hours. Next, decrease the temperature to $80^{\circ} \mathrm{C}$ and pour in the previously prepared solution mixture of $5 \mathrm{ml}$ OLA and $2.5 \mathrm{ml}$ ODE, stir vigorously for $30 \mathrm{~min}$. Finally, decreases the temperature to $40^{\circ} \mathrm{C}$, we obtain Cd precursor solution in medium solution of OLA-ODE.

Preparing TOP-Se precursor: weigh 0,04 g Se powder, respective to $0.5 \mathrm{mmol}$, put into a flask, then pour in this flask 0.5 $\mathrm{ml}$ TOP. Vacuum all the air from the flask, pump in $\mathrm{N}_{2}$ gas continuously. Put that flask into ultrasonic vibrator in water at $80-100^{\circ} \mathrm{C}$ until Se dissolves completely, which is about 10 minutes.

Preparing TOP-Te precursor: weight $0,064 \mathrm{~g}$ Te powder, respective to $0.5 \mathrm{mmol}$, put into a flask, and then pour in the flask $0.85 \mathrm{ml}$ TOP. Proceed the same as above. However, since Te is a metal powder that is hard to dissolve in TOP, we need to vacuum carefully to remove all the air in the flask for a very long period and before running $\mathrm{N}_{2}$ gas through it. Put this mixture into hot water $\left(80^{\circ} \mathrm{C}-90^{\circ} \mathrm{C}\right)$ in ultrasonic vibrator, until Te dissolves completely, which takes about 15 minutes.

Inject TOP-Se solution into a flask with TOP-Te, mix by ultrasonic vibrator in $15 \mathrm{~min}$. to allow these two precursors to be completely mixed, and then we obtain TOP-Se and TOP-Te to be used in alloy QD fabrication.

*CdTeSe core quantum dots synthesis: the solution mixtures TOP-Se and TOP-Te fabricated above are fast-injected into a three-neck flask with Cd precursor solution, at $120^{\circ} \mathrm{C}$ in $1 \mathrm{~h}$, in $\mathrm{N}_{2}$ gas. Afterwards, the solution temperature increases, until it reaches $180^{\circ} \mathrm{C}, 200^{\circ} \mathrm{C}$ or $220^{\circ} \mathrm{C}$ (different samples were synthesized in order to analyze the effect of the growth temperature). Keep the temperature for $10 \mathrm{~min}$ and mix vigorously with magnetic stirrerso that the nano particles are grown. At this temperature, the original solution is red, and then gradually changes to dark brown. Next, turn off the heater; let the solution cool down while still stirring it with magnetic stirrer. The volume of obtained CdTeSe solution is $\sim 85 \mathrm{ml}$.

*CdTeSe/ZnSe and CdTeSe/ZnTecore/shell quantum dots synthesis

Preparing the precursors for the shell:other than the precursors of Te and Se being prepared like the precursor for the core, we also need to prepare the precursors of $\mathrm{Cd}$ and $\mathrm{Zn}$.

In order to fabricate the cadmium stock solution, we dissolved $0.025 \mathrm{~g}$ of cadmium acetatein $0.54 \mathrm{ml} \mathrm{TOP}$ at $80^{\circ} \mathrm{C}$ in nitrogen gas. Similarly, we obtained the zinc stock solution by dissolving $0.28 \mathrm{~g}$ of zinc acetate in $4.2 \mathrm{ml}$ TOP in a flask, at $120^{\circ} \mathrm{C}$ in nitrogen gas, until it was completely dissolved, which took around 30 mins.

The weight of $\mathrm{Zn}$ and Te were calculated for $1 \mathrm{ML}, 2 \mathrm{ML}, 4 \mathrm{ML}$ and $6 \mathrm{ML}$ of $\mathrm{ZnSe}$ and ZnTe. A monolayer thickness is based on the lattice constant a of ZnSe or ZnTe crystals, depending on the type of shell. The molar ratio of Zn: Te was 1:1.

Coating with shell: Put $46.4 \mathrm{ml}$ of CdTeSe core QDs $(\sim 1.6 \mathrm{mmol})$ into a three-neck flask, and quickly raise the temperature to the lower temperature than the core growth temperature $\sim 20^{\circ} \mathrm{C}$. At this temperature, quickly inject $2.8 \mathrm{ml}$ of $\mathrm{Zn}$ precursor solution (respective to a monolayer of Zn ions), stir vigorously in $15 \mathrm{~min}$. Then, quickly inject $1.3 \mathrm{ml}$ of TOP-Te in to grow the particles' shell in $15 \mathrm{~min}$. Next, take out $25 \mathrm{ml}$ of solution containing QDs, which is CdTeSe/ZnTe 1ML. With the remaining volume, we continue to quickly inject $1.4 \mathrm{ml}$ of $\mathrm{Zn}$ precursor, stir vigorously in $10 \mathrm{~min}$, then TOP-Se $(0.7 \mathrm{ml})$, stir vigorously to grow the ZnTe particles' shell in $15 \mathrm{~min}$. We obtain CdTeSe/ZnTe $2 \mathrm{ML}$. Do the same when coating $\mathrm{ZnSe}$ for CdTeSe QD cores to form CdTeSe/ZnSe.

All ternary quantum dots were purified by several rounds of precipitation and centrifugation and were stored at room temperature for later characterization and use.

\subsection{Characterization of CdTeSe /ZnSe (Te) core/shell ternary quantum dots}

The size of the core QDs and the shell thickness were determined by the transmission electron microscopy (TEM) with a JEOL Jem 1010 microscope operating at $100 \mathrm{kV}$. Powder X-ray diffraction (XRD, Siemens D5005) was used to confirm the wurtzite $(\mathrm{w})$ or zinc-blende (zb) crystalline structure. The XRD patterns were compared with the tabulated values of bulk CdSe (JCPDS card No. 19-191 (zb) and 8-459 (w)),CdTe: 15-770 (zb), 19-193 (w), ZnSe (JCPDS 37-1463 (zb) and 15- 105 (w),ZnTe: 15-746 (zb), 19-1482 (w) and CdTeSe (w):41-1325. 
The UV-Vis absorption spectra of QDs in toluen were scanned within the wavelength range of 200-600 $\mathrm{nm}$ using a Shimadzu (UV-1800) UV-Vis spectrophotometer. All UV-Vis measurements were performed at $25^{\circ} \mathrm{C}$ and automatically corrected for the solvent medium, which was the toluene.

Fluorescence spectra measurement is carried out on the Fluorolog-322 system by Horiba Jobin Yvon using Xenon 450W light, the detector is a photomultiplier, measuring range from 250-800nm. Acton SpectraPro-2300i spectrometer with $\mathrm{He}-$ $\mathrm{Cd}$ laser emitted at two wavelengths $442 \mathrm{~nm}$ and $325 \mathrm{~nm}$ also was used to measure emission spectra. The PL decays were analyzed with a PM Hamamatsu R5600U and a Tektronix TDS 784A scope with a time resolution of $1 \mathrm{~ns}$.

The QDs samples were analyzed by Micro Raman spectroscopy (XploRA- Horiba) using $532 \mathrm{~nm}(90 \mathrm{~mW})$ or $785 \mathrm{~nm}(25$ $\mathrm{mW}$ ) excitation line from a diode pumped, solid state laser to analyze the vibration bonds and their Raman frequencies. The laser power was $100 \mathrm{~mW}$. 10× Objectives were used to focus the excitation laser light on the right spot of the investigated samples. The spot size of laser beam was $1 \mu \mathrm{m}$. The spectral resolution was $2 \mathrm{~cm}^{-1}$. The acquisition time was from $30 \mathrm{~s}$ to 120 , but normally was $30 \mathrm{~s}$. The system uses CCD receiver with four gratings $600,1200,1800$ and $2400 \mathrm{gr} . / \mathrm{mm}$, measuring range from $100-4000 \mathrm{~cm}^{-1}$.

\section{Results and Discussion}

The aim of this research is to fabricate CdTeSe QDs, whose emission can change in the range from red to near infrared (NIR), to apply in sensitizers for solar cells (Quantum Dot Sensitized Solar Cells - QDSC) or biology. This study was also conducted to find out the method that uses little of TOP and none of trioctylphosphine oxide (TOPO) and hexadecylamine (HDA), and grows QDs at a moderate temperature $\left(\sim 220^{\circ} \mathrm{C}\right)$. To eliminate the electronic traps on the surface of the QDs and make it easy to modify and functionalize their surfaces, the quantum dots are coated. Two kinds of different shell materials have been used, which are ZnTe and ZnSe. Here are some experimentalresults on the CdTeSe cores fabricated under these conditions: two different molar ratios used, crystal growth medium, growth temperature, etc., and the results on QDs with core/shell structure.

\subsection{TEM images}

All fabricated samples had their TEM images recorded, but we only get clear TEM images of some samples. Figure 1 presents the TEM images of samples CdTeSe QDs prepared at $180^{\circ} \mathrm{C}, 200^{\circ} \mathrm{C}$ and $220^{\circ} \mathrm{C}(\mathrm{N} 4-15)$, the samples CdTeSe/ZnSe nML ( $\mathrm{n}=$ 0,2 and 4) (N11-15) and the samples CdTeSe/ZnTe nML (n = 0, 2 and 4) (N12-15). The shape of these QDs is rather elongated. The long-axis diameter of the CdTeSe QDs is $5.3 \mathrm{~nm}$ for the $180^{\circ} \mathrm{C}$ growth, is $5.8 \mathrm{~nm}$ for $200^{\circ} \mathrm{C}$ and $6.4 \mathrm{~nm}$ for $220^{\circ} \mathrm{C}$ (series N4-15). The size of QDs is for samples series N11-15: $6.9 \mathrm{~nm}$ for the core, $7.7 \mathrm{~nm}$ when coated with an additional 2ML ZnSe shell (in longer dimension), and 8.2 nm with 4ML. For the series N12-15: the core size is $7 \mathrm{~nm}, 7.4 \mathrm{~nm}$ with ZnTe $2 \mathrm{ML}$ and $7.8 \mathrm{~nm}$ with 4ML. The shorter dimension reaches approximately $\sim 5 \mathrm{~nm}$.

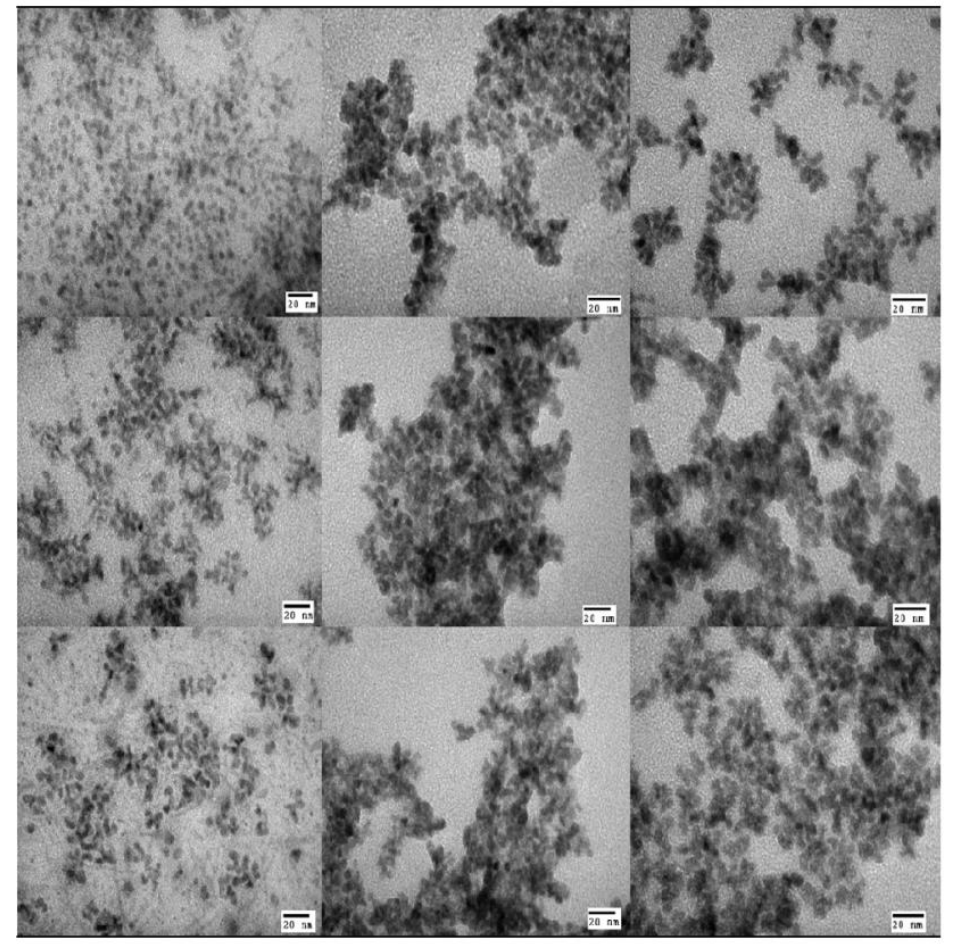

Fig. 1. TEM imagesof (left) the CdTeSe QDs prepared at $180^{\circ} \mathrm{C}, 200^{\circ} \mathrm{C}$ and $220^{\circ} \mathrm{C}$, from top to bottom (N4-15), (middle) the samples CdTeSe/ZnSe nML ( $\mathrm{n}=0,2$ and 4) (N11-15) and (right) the samples CdTeSe/ZnTe nML ( $\mathrm{n}=0,2$ and 4) (N1215), from top to bottom. Scale bars: $20 \mathrm{~nm}$.

\subsection{Raman spectra}




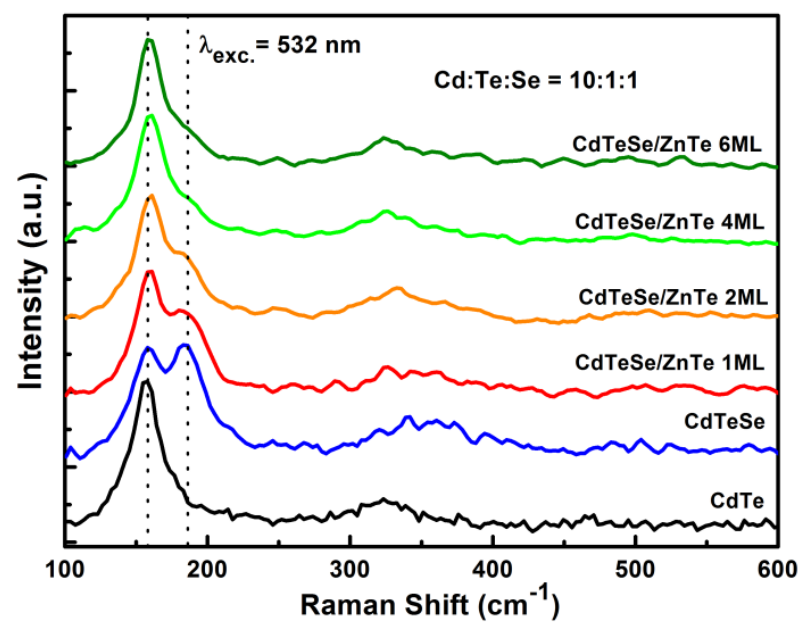

Fig. 2. Raman spectra of CdTeSe and CdTeSe/ZnTe nML ( $\mathrm{n}=1,2,4$ and 6) (N12-15) QDs.

We used the information on the phonon spectrum provided by Raman spectroscopy in order to analyze thecomposition of CdTeSe QDs coated with ZnTe and ZnSe, forming CdTeSe/ZnTe and CdTeSe/ZnSe core/shell structures. Fig. 2 shows the Raman spectra of the series of CdTeSe coated with ZnTe, when the shell thickness changes from 1 ML to 6 ML. In this figure, the Raman spectrum of CdTe is brought in to be referred and compared to the Raman spectra of the QD samples presented in this research. The peak around $157 \mathrm{~cm}^{-1}$ is characteristic of CdTe longitudinal optical (LO) phonon [15, 16], and its two-phonon replica is also seen weakly at $315 \mathrm{~cm}^{-1}$. The spectrum of the CdTeSe cores show a second peak at 192 $\mathrm{cm}^{-1}$, which corresponds to the characteristic vibration of the CdTeSe alloy [16-18]. When CdTeSe is coated with a ZnTe monolayer, we observe a similar spectrum: the frequency position of the first peak lies at $159 \mathrm{~cm}^{-1}$ and that of the second peak lies at $184 \mathrm{~cm}^{-1}$. The intensity of the peak at $159 \mathrm{~cm}^{-1}$ is stronger compared to the peak at $184 \mathrm{~cm}^{-1}$. However, when the shell thickness increases from 2 to $6 \mathrm{ML}$, only one peak remains at $159 \mathrm{~cm}^{-1}$ (again with a two-phonon replica at 315 $\mathrm{cm}^{-1}$ ) the other peak appears as a shoulder which decreases as the shell thickness increases. These results suggest that when the ZnTe shell thickness is increased above $2 \mathrm{ML}$, the CdTeSe ternary alloy QDs become CdTe-rich QDs. This may be explained by the strong chemical activity of the Te element, so that when a large amount is brought into the reaction flask for the shell growth, it immediately reacts to the abundant $\mathrm{Cd}$ ions from the CdTeSe core fabrication (the $\mathrm{Cd}$ molar ratio is 5 times larger than Te and Se), to create a CdTe layer around CdTeSe.

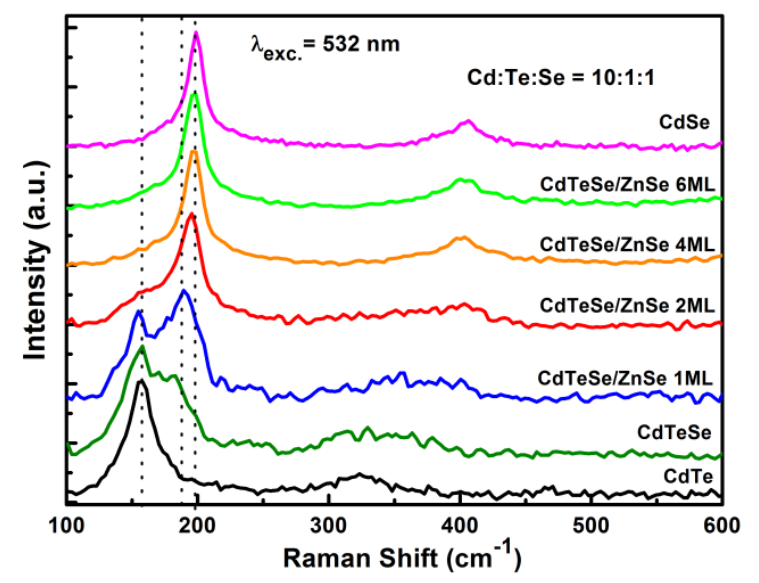

Fig.3. Raman spectra of the series CdTeSe/ZnSe $\mathrm{nML}$ ( $\mathrm{n}=0$, 1, 2, 4 and 6) QDs samples (N11-15)

When the CdTeSe QDs are coated with ZnSe shell from 2 ML to 6 ML thickness to form core/shell QDs, we can observe a similar phenomenon, but this time it is the characteristic line of the CdSe vibration which increases. Fig. 3 shows the Raman spectra of the CdTeSe/ZnSe nML ( $\mathrm{n}=0,1,2,4$ and 6) series. On the Raman spectra, there are two observable peaks at $158 \mathrm{~cm}^{-1}$ and $188 \mathrm{~cm}^{-1}$ of the CdTeSe core and CdTeSe/ZnSe 1ML. These lines are characteristic of the vibration 
of the ternary alloy CdSeTe QD phase, as discussed previously. When the nominal shell thickness increases above $2 \mathrm{ML}$, a vibration line at $200 \mathrm{~cm}^{-1}$ appears and prevails, which can be assigned to the LO peak of CdSe $\left(200 \mathrm{~cm}^{-1}\right)$. This result suggests that, when the $\mathrm{Zn}$ and Se precursors are introduced for the shell growth, since excess $\mathrm{Cd}$ ions are still present while all Te ions have reacted, in this case, a CdSe material layer forms gradually on the CdTeSe core, thus we obtain CdSe-rich QDs.

\subsection{XRD data}

The results on crystalline phase analysis by the powder X-ray diffraction (XRD) method show that, depending on the fabricating conditions and different Cd: Te: Se molar ratios, the CdTeSe/ZnTe and CdTeSe/ZnSe QDs show up at different crystalline phases. For the core and core-shell samples, the XRD data (Figs. 4, 5), although broadened due to the finite size of the nanocrystallites, evidence the zinc-blende type of the crystalline structure: they exhibit the three peaks (a singlet peak at low angle and a doublet of peaks at high angle) characteristic of the zinc-blende patterns, whereas the wurtzite patterns have four peaks (a singlet at low angle and a triplet at high angle) $[1,19,20]$.

For the cores CdTeSe prepared at different temperatures or Cd:Te:Se ratios, (Fig. 4), we can observe on the diffraction diagram the characteristic peaks located between the crystalline phase CdTe (zb) and $\mathrm{CdSe}(\mathrm{zb})$. Therefore we can assume that the QDs have crystallized into zb CdTeSe crystals in the fabricated samples. The peaks are generally slightly closer to the zb-CdTe lines than to the zb-CdSe lines, which would indicate a Te-rich alloy, in agreement with Raman data.

Fig. 5 presents the X-ray diffraction patterns of the core-shell CdTeSe/ZnSe sample series. The XRD spectrum is not changed when coating of $\mathrm{ZnSe}$ at $1 \mathrm{ML}$. However, when the ZnSe shell thickness reaches 2 or $4 \mathrm{ML}$, the XRD peaks are broadened, possibly due to sample inhomogeneities or to non-uniform crystalline phases inside a QD, and shifted. The positions of the peaks for the $4 \mathrm{ML}$ sample are shifted towards the tabulated ZnSe peaks positions, however their proximity to the peaks of CdSe might also reflect the presence of CdSe indicated by the Raman spectra.

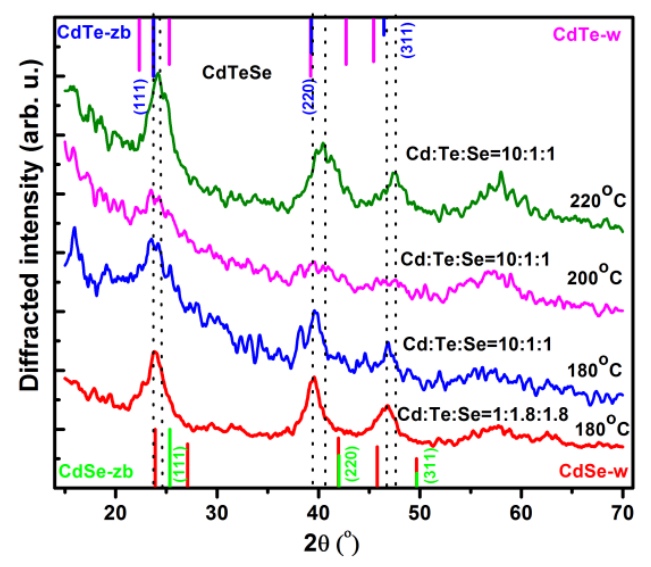

Fig. 4. Powder XRD patterns of CdTeSe ternary QD cores (sample N4) prepared at different temperatures at $180^{\circ} \mathrm{C}$ (for Cd: Te: $\mathrm{Se}=10: 1: 1$ and $1: 1.8: 1.8), 200^{\circ} \mathrm{C}$ and $220^{\circ} \mathrm{C}$. The tabulated values of the bulk diffraction peaks for wurtzite $(\mathrm{w})$ and zinc blend (zb) CdTe (top) and CdSe (bottom) are shown.

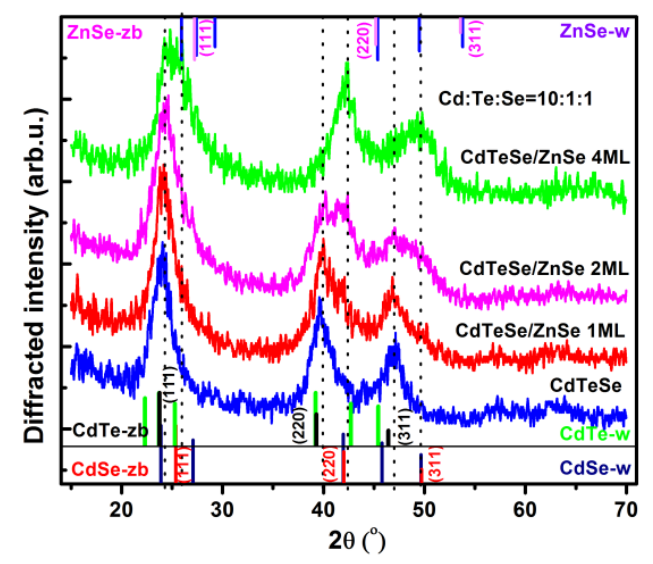

Fig. 5. Powder XRD patterns of ternary core/shell QDs CdTeSe/ZnSe $n M L ~\left(n=0,1,2\right.$ and 4)prepared at $180^{\circ} \mathrm{C}(10 \mathrm{~min}$.). The tabulated positions of the bulk diffraction peaks for wurtzite (w) and zinc blend (zb) CdSe (bottom) and CdTe (middle) and $\mathrm{ZnSe}$ (top) are shown. 
Fig. 6 shows the absorption spectra and normalized photoluminescence (PL) spectra of two samples of alloyed CdSeTe core QDs that we fabricated, with two different molar ratios: Cd:Te:Se $=1: 1.8: 1.8$ and 10:1:1, as noted on the figure. The absorption spectra display a clear exciton peak showing high quality of the QDs. However, the QD samples fabricated with the ratio $\mathrm{Cd}$ : $\mathrm{Te}: \mathrm{Se}=1: 1.8: 1.8$ has clearer and sharper exciton peaks. The QD emission wavelength ranges from $650 \mathrm{~nm}$ to $700 \mathrm{~nm}$, different on each sample, it could depend on both the alloy band gap and on the QD diameter . However, given the similar sizes of these samples, we expect that most contribution to the optical transition energy comes from the change in the QDs' compositions $(\mathrm{Cd} / \mathrm{Te}+\mathrm{Se})$ ratio). One can see also the larger Stokes shifts in samples that may originate from the localization of charge carriers in the alloy, as similarly observed in $\operatorname{In}(\mathrm{Zn}) \mathrm{P}$ [21] and CdZnSe QDs [13].

Fig.7 shows the PL decay curve, for two CdTeSe QD samples: N3-0-14 and N4-0-14. These curves are slightly multiexponential, with a typical decay time (measured at $1 / \mathrm{e}$ decay) $\mathrm{t}=41 \mathrm{~ns}(\mathrm{~N} 3-0-14)$ and $\mathrm{t}=43 \mathrm{~ns}(\mathrm{~N} 4-0-14)$. These values are of the same order and suitable with the lifetime values reported in [22]. The absence of any short-lived component is a strong indication of the weakness of the non-radiative decay contributions and suggests rather high quantum efficiencies.

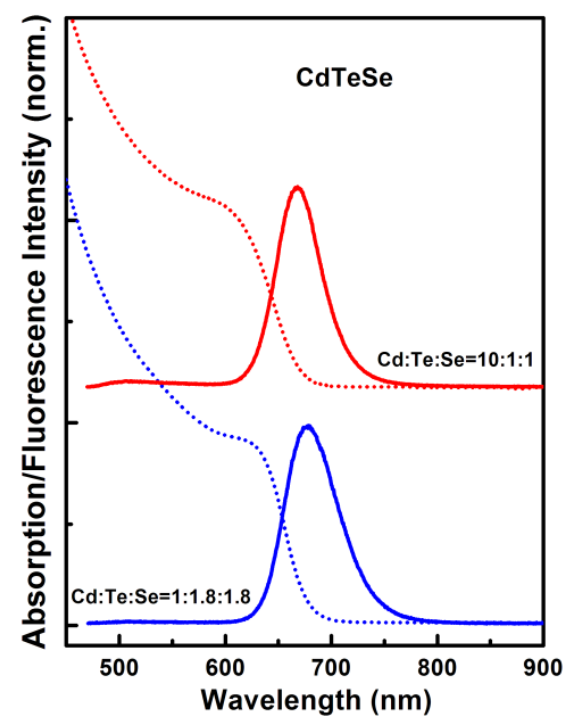

Fig. 6. Absorption (dotted lines) and normalized photoluminescence (full lines) spectra of the CdTeSe core samples prepared by two different molar ratios (norm. units).

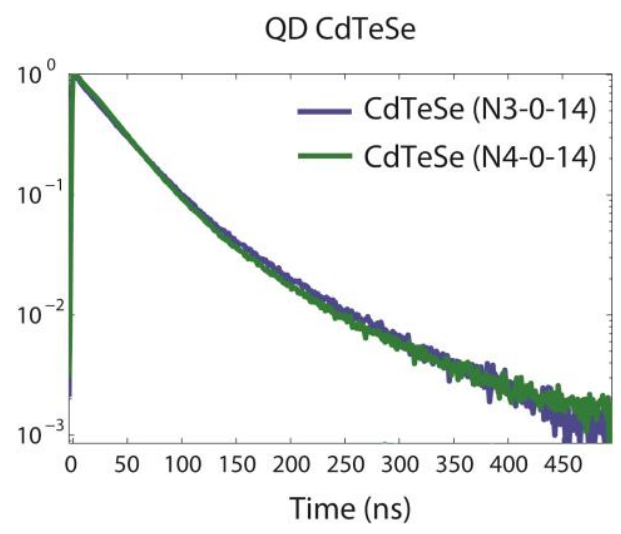

Fig.7. PL decay curves (in ln scale) of the samples CdTeSe N3 (blue) and N4 (green). The lifetime (measured at 1/e decay) of the CdTeSe core quantum dots $\tau$ CdTeSe $(\mathrm{N} 3-0-14)=41 \mathrm{~ns} \tau$ CdTeSe $(\mathrm{N} 4-0-14)=43 \mathrm{~ns}$.

We have also fabricated CdTeSe QD samples with emission band at $828 \mathrm{~nm}$, and coated with ZnSe shells up to 6ML thick. When coated with $\mathrm{ZnSe}$, the absorption and emission band (Fig. 8) shifts towards the longer wavelengths, increasing with the thickness of ZnSe. The emission peak of these QDs reaches $866 \mathrm{~nm}$ at $1 \mathrm{ML}, 915 \mathrm{~nm}$ at $2 \mathrm{ML}, 925 \mathrm{~nm}$ at $4 \mathrm{ML}$ and 940 $\mathrm{nm}$ at 6ML.The reason for this shift is not yet fully understood; it may involved decay through surface traps created at the shell surface. The emission intensity increases when coated with ZnSe 1ML and 2ML. However, when the thickness 
reaches 4ML, the emission intensity decreases. Therefore, it can be said that, for CdTeSe QDs, the optimum ZnSe-shell thickness is 2ML. The measurement of the life time of these QD samples (not shown here) also shows that, when CdTeSe is coated with a 1 or $2 \mathrm{ML}$ layer of $\mathrm{ZnSe}$, its life time is longer than the core's. This matches the results on the increase of emission intensity when the shell reaches $2 \mathrm{ML}$ of $\mathrm{ZnSe}$.

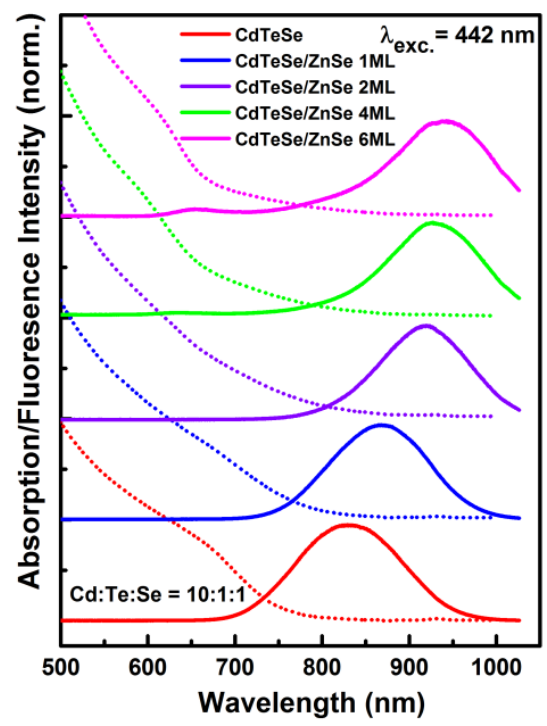

Fig. 8. Absorption (dotted lines) and photoluminescence (full lines) spectra of the five CdTeSe/ZnSe nML, $\mathrm{n}=0,1,2,4$ and 6 . (norm. units). $\mathrm{T}=220^{\circ} \mathrm{C}\left(10 \mathrm{~min}\right.$.) $\mathrm{T}$ shell $=200^{\circ} \mathrm{C}(10 \mathrm{~min}$. $)$.

\section{Conclusion}

In summary, we have successfully fabricated CdTeSe QDs with core/shell structure with the molar ratio Cd: Te: Se = 10:1:1, at temperatures from 180 to $220^{\circ} \mathrm{C}$. The use of $\mathrm{ZnSe}$ and $\mathrm{ZnTe}$ allowed protection of the core. These core/shell CdTeSe QDs have an elongated shape, with size $\sim 8 \mathrm{~nm}$, changing depending on each sample. The characterization of these QDs with Raman spectroscopy has shown that it is a strong tool to detect the forming of the ternary alloyed CdTeSe crystalline phase. This research shows that the best thickness of the ZnSe or ZnTe shell for the CdTeSe QDs' core is 2 ML and that some incorporation of the Se or Te inside the core might occur. The QDs with core/shell structure like $\mathrm{CdTeSe} / \mathrm{ZnSe}$ can absorb up to nearly $800 \mathrm{~nm}$ and emit up to nearly $900 \mathrm{~nm}$. We are presently working on the application of these QDs to improve NIR absorption of solar cell devices.

\section{Acknowledgments}

This research is funded by Vietnam National Foundation for Science and Technology Development (NAFOSTED) under grant number 103.03-2014.66, the PICS cooperation projects between CNRS and VAST (Project Number 6456 and VAST.HTQT. Phap. 01/15-16), by the Centre de Compétences C'Nano- Ile de France (NanoPlasmAA project) and the Agence Nationale de la Recherche (Ponimi project). The authors thank the National Key Laboratory for Electronic Materials and Devices - IMS and Duy Tan University for the use of facilities.

\section{References}

[1] R.E. Bailey, S. Nie, J. Am. Chem. Soc. 125, 7100 (2003).

[2] T. Pons, N. Lequeux, B. Mahler, S. Sasnouski, A. Fragola, B. Dubertret, Chem Mater. 21, 1418 (2009).

[3] P. Yang, S. Wang and N. Murase, Nanoscale Research Letters 7, 615 (2012).

[4] J.L. C. Espinola, T. V. Torchynska, J. A. J. Gómez, J. Douda and K. Gazarian, Mater. Res. Soc. Symp. Proc., Vol. 1534, (2013).

[5] T.V.Torchynsk, Physica E 51, 55 (2013).

[6] G. X. Liang, L. L. Li, H. Y. Liu, J. R. Zhang, C. Burda and . J. Zhu, Chem. Commun. 46, 2974 (2010).

[7] X. Xu, Y. Wang, W. Xia, L. Zhou, F. Gong, L. Wu, Mater. Chem. and Phys. 139, 210 (2013).

[8] H. Zhou, G. Zhou, J. Zhou, D. Xu, X. Zhang, P. Kong, Z. Yang, Mater. Res. Bull., 65, 53 (2015).

[9] R. Wang, O. Calvignanello, C. I. Ratcliffe,X. Wu,D. M. Leek, Md. B. Zaman, D. Kingston,J. A. Ripmeester, and K. Yu, J. Phys. Chem. C 113, 3402 (2009).

[10] L. Liu, X. Xu, T. Luo, Y. Liu, Z. Yang, J. Lei, Solid State Com. 152, 1103 (2012).

[11] L. Liao, H. Zhang, X. Zhong, J. Luminescence 131, 322 (2011).

[12] F. Yang, Z. Xu, J. Wang, F. Zan, C. Dong and J. Ren, J. Luminescence 28, 392 (2013). 
[13] N. H. Yen, W. D. D. Marcillac,C. Lethiec, P. N. Hong, C. Schwob, A. Maître, N. Q. Liem, L. V. Vu, P. Bénalloul, L. Coolen and P. T. Nga, Opt. Mater. 36, 1534 (2014).

[14] Z. Pan, K. Zhao, J. Wang, H. Zhang, Y. Feng, and X. Zhong, ACS Nano, 7,5215 (2013).

[15] V. Dzhagan, I. Lokteva, C. Himcinschi, X. Jin, J. K. Olesiak, D. RT Zahn, Nanoscale Res. Lett. 6, 79 (2011).

[16] S. Li., G. Tan., J. B. Murowchick.,C. Wisner., N. Leventis, T. Xia, X. Chen., and Z. Peng, J. Electron Mater. 42 , No. 12, 3373 (2013).

[17 ] B. T. Spann and X. Xu, Appl. Phys. Lett. 105, 083111 (2014).

[18] Z. Chai, W. Wu, D. Kong, Y. Gao, and Q. Chang, J. Non-Cryst. Solids 82, 121 (2013).

[19] S. H.Tolbert, A. P. Alivisatos, A. P. Science 265, 373 (1994).

[20] S. H.Tolbert, A. P. Alivisatos, Annu. Rev. Phys. Chem. 46, 595 (1995).

[21] U. T. D. Thuy, P.Reiss, and N. Q. Liem, Appl. Phys. Lett. 97, 193104 (2010).

[22] L. Li, Y. Chen, Q. Lu, J. Ji, Y. Shen, M. Xu, R. Fei, G. Yang, K. Zhang, J. R. Zhang \& J. J. Zhu, Sci Rep. 3 , 1529 (2013). 